EPSC Abstracts

\title{
Measuring and modeling the Balmer series in hot gaseous giant exoplanets
}

\author{
Aurélien Wyttenbach ${ }^{1,2}$, Paul Mollière ${ }^{2,3}$, David Ehrenreich ${ }^{4}$, Heather Cegla ${ }^{4}$, Vincent Bourrier ${ }^{4}$, \\ Christophe Lovis ${ }^{4}$, Lorenzo Pino ${ }^{5}$, Romain Allart ${ }^{4}$, Julia Seidel ${ }^{4}$, Jens Hoeijmakers ${ }^{4,6}$, Louise Nielsen ${ }^{4}$, \\ Baptiste Lavie ${ }^{4}$, Francesco Pepe ${ }^{4}$, Xavier Bonfils ${ }^{1}$, and Ignas Snellen ${ }^{2}$ \\ ${ }^{1}$ Université Grenoble Alpes, CNRS, IPAG, 38000 Grenoble, France (aurelienwyttenbach@gmail.com) \\ ${ }^{2}$ Leiden Observatory, Leiden University, Postbus 9513, 2300 RA Leiden, The Netherlands \\ ${ }^{3}$ Max-Planck-Institut für Astronomie, Königstuhl 17, 69117 Heidelberg, Germany \\ ${ }^{4}$ Geneva Observatory, University of Geneva, Ch. des Maillettes 51, 1290 Versoix, Switzerland \\ ${ }^{5}$ Anton Pannekoek Institute for Astronomy, University of Amsterdam, Science Park 904, 1098 XH Amsterdam, The Netherlands \\ ${ }^{6}$ University of Bern, Center for Space and Habitability, Sidlerstrasse 5, 3012 Bern, Switzerland
}

\begin{abstract}
Atmospheric escape rate is a key parameter to measure in order to understand the evolution of exoplanets. In this presentation, we will show that the Balmer series, observed with high-resolution transmission spectroscopy, is a precise probe to measure exoplanet evaporation, especially for ultra hot Jupiters orbiting early-type star. These hot gaseous giant exoplanets (such as KELT-9 b) are presumed to have an atmosphere dominated by neutral and ionized atomic species. In particular, hydrogen Balmer lines have been detected in some of their upper atmospheres, suggesting that hydrogen is filling the planetary Roche lobe and escaping from these planets. Here, we will present new significant absorptions of the Balmer series in the KELT-9b atmosphere obtained with HARPS-N. The precise line shapes of the $\mathrm{Ha}, \mathrm{H} \beta$, and $\mathrm{Hy}$ absorptions allow us to put constraints on the thermospheric temperature. Moreover, the mass loss rate, and the excited hydrogen population of KELT-9 $b$ are also constrained, thanks to a retrieval analysis performed with a new atmospheric model (the PAWN model). We retrieved a thermospheric temperature of $\mathrm{T}=13200+800-720 \mathrm{~K}$ and a mass loss rate of $\log 10(M L R)=10^{\wedge}(12.8+-0.3) \mathrm{g} / \mathrm{s}$ when the atmosphere was assumed to be in hydrodynamical expansion and in local thermodynamic equilibrium (LTE). Since the thermospheres of hot Jupiters are not expected to be in LTE, we explored atmospheric structures with nonBoltzmann equilibrium for the population of the excited hydrogen. We do not find strong statistical evidence in favor of a departure from LTE. However, our non-LTE scenario suggests that a departure from the Boltzmann equilibrium may not be sufficient to explain the retrieved low number densities of the excited hydrogen. In non-LTE, Saha equilibrium departure via photo-ionization, is also likely to be necessary to explain the data.
\end{abstract}

